\title{
P05-09. 4e I 0 epitope-scaffolds mimic the antibody-bound epitope conformation and block neutralization by sera from rare HIV+ individuals
}

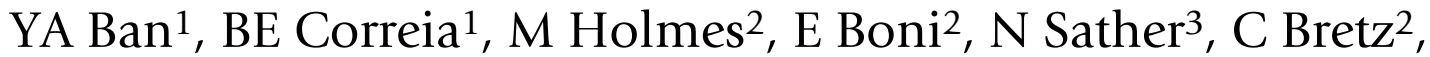 \\ O Kalyuzhniy ${ }^{1}, \mathrm{C} \mathrm{Xu}^{2}$, D Baker ${ }^{1}$, L Stamatatos ${ }^{3}$, R Strong ${ }^{2}$ and W Schief*1
}

Address: ${ }^{1}$ Biochemistry, University of Washington, Seattle, WA, USA, ${ }^{2}$ Fred Hutchinson Cancer Research Center, Seattle, WA, USA and ${ }^{3}$ Seattle Biomedical Research Institute, Seattle, USA

* Corresponding author

from AIDS Vaccine 2009

Paris, France. 19-22 October 2009

Published: 22 October 2009

Retrovirology 2009, 6(Suppl 3):P85 doi:I0.1 I86/1742-4690-6-S3-P85

This abstract is available from: http://www.retrovirology.com/content/6/S3/P85

(c) 2009 Ban et al; licensee BioMed Central Ltd.

\section{Background}

A protective vaccine against HIV will likely require elicitation of potent broadly neutralizing antibodies as well as cellular immunity. mAb $4 \mathrm{e} 10$ has the greatest breadth of the known neutralizing monoclonal antibodies, but 4e10-like antibodies are rarely elicited in natural infection. The crystal structure of the $4 \mathrm{e} 10$ antibody-epitope complex was solved previously. To develop immunogens that elicit 4e10-like antibodies, we employed computational methods to design " $4 \mathrm{e} 10$ epitope-scaffolds" - protein scaffolds that present and stabilize the 4e10-bound conformation of the epitope.

\section{Methods}

Within the Rosetta protein modeling platform we developed methods that (1) identified protein scaffolds with structural similarity to the 4e10 epitope and suitable for $4 \mathrm{e} 10$ binding without steric clash; and (2) transplanted epitope side-chains to scaffolds and optimized the scaffold-antibody interaction. Epitope-scaffolds were designed automatically and then subjected to humanguided refinement.

\section{Results}

More than twenty epitope-scaffolds were expressed and purified. Most of the epitope-scaffolds bound to $4 \mathrm{e} 10$ with 10- to 1000-fold higher affinity than the cognate peptide, as assessed by surface plasmon resonance. Crystal structures of multiple epitope-scaffolds were determined, both unbound (six structures) and bound (two structures) to the $4 \mathrm{e} 10$ antibody. Multiple bound and unbound structures revealed near perfect mimicry of the $4 \mathrm{e} 10$ epitope conformation. All the epitope-scaffolds tested blocked broadly-neutralizing activity in serum from an HIV-1 chronically-infected individual who developed 4E10-like NAbs; one scaffold mutated to kill the epitope did not block this activity, indicating that the block was specific to the epitope.

\section{Conclusion}

We successfully transferred the $4 \mathrm{e} 10$ epitope to non-HIV protein scaffolds. The scaffolds are useful as candidate immunogens and as reagents to characterize cross-reactive neutralizing specificities in HIV+ sera. The immunogen design methods are general and can be applied to other epitopes with other conformations.

\section{Acknowledgmenet}

This research was conducted as part of the Collaboration for AIDS Vaccine Discovery with support from the Bill \& Melinda Gates Foundation. 